\title{
Disorders of consciousness: further pathophysiological insights using motor cortex transcranial magnetic stimulation
}

\author{
Natallia Lapitskaya ${ }^{1,2, *}$, Martin R. Coleman ${ }^{3}$, Joergen Feldbaek Nielsen ${ }^{1}$, \\ Olivia Gosseries ${ }^{2}$ and Alain Maertens de Noordhout ${ }^{4}$ \\ ${ }^{1}$ Neurorehabilitation Research Department, Hammel Neurorehabilitation and Research Centre, \\ Hammel, Denmark \\ ${ }^{2}$ Coma Science Group, Cyclotron Research Centre, University of Liège, Liège, Belgium \\ ${ }^{3}$ Impaired Consciousness Research Group, Wolfson Brain Imaging Centre, University of Cambridge, UK \\ ${ }^{4}$ Neurology Department, University of Liège, Centre Hospitalier Regional de la Citadelle, Liège, Belgium
}

\begin{abstract}
Transcranial magnetic stimulation (TMS) is a noninvasive means of investigating the function, plasticity, and excitability of the human brain. TMS induces a brief intracranial electrical current, which produces action potentials in excitable cells. Stimulation applied over the motor cortex can be used to measure overall excitability of the corticospinal system, somatotopic representation of muscles, and subsequent plastic changes following injury. The facilitation and inhibition characteristics of the cerebral cortex can also be compared using the modulatory effect of a conditioning stimulus preceding a test stimulus. So called paired-pulse protocols have been used in humans and animals to assess GABA $(\gamma$-amino-butyric acid)-ergic function and may have a future role directing therapeutic interventions. Indeed, repetitive magnetic stimulation, where intracranial currents are induced by repetitive stimulation higher than $1 \mathrm{~Hz}$, has been shown to modulate brain responses to sensory and cognitive stimulation. Here, we summarize information gathered using TMS with patients in coma, vegetative state, and minimally conscious state. Although in the early stages of investigation, there is preliminary evidence that TMS represents a promising tool by which to elucidate the pathophysiological sequelae of impaired consciousness and potentially direct future therapeutic interventions. We will discuss the methodology of work conducted to date, as well as debate the general limitations and pitfalls of TMS studies in patients with altered states of consciousness.
\end{abstract}

Keywords: coma; vegetative state; minimally conscious state; brain injury; transcranial magnetic stimulation; repetitive transcranial magnetic stimulation

\section{Introduction}

*Corresponding author.

Tel.: +45 8762 3573; Fax: +45 8762 3305;

E-mail: natallia.lapitskaya@hammel.rm.dk
Over the past two decades, transcranial magnetic stimulation (TMS) has provided fascinating insight into the mechanisms and consequences of 
cortical plasticity in the intact and damaged human brain (e.g., see Siebner and Rothwell, 2003). However, very few studies have focused on severe brain injury and disorders of consciousness (DOC), specifically, the conditions of coma, vegetative state (VS), and minimally conscious state (MCS). This chapter provides a brief introduction to the technique of TMS, before summarizing information gathered using TMS in DOC. Although in the early stages of investigation, there is preliminary evidence to suggest that TMS represents a promising tool by which to elucidate the pathophysiological sequelae of impaired consciousness and potentially direct future therapeutic interventions. We will discuss the methodology of work conducted to date, as well as debate the general limitations and pitfalls of TMS studies in patients with DOC. Finally, we will also suggest possible areas of future TMS investigation with these challenging patients.

\section{General principles of TMS}

TMS represents a noninvasive, generally pain free, means of stimulating the cerebral cortex (Barker and Jalinous, 1985). In classic TMS experiments, a plastic-coated coil of wire is placed over the scalp, through which a powerful and rapidly changing current is passed, to create a magnetic field that penetrates the cranium and neuronal membrane, to produce excitatory or inhibitory postsynaptic potentials (reviewed in Di Lazzaro et al., 2004). In most experiments, stimulation is delivered to the primary motor cortex, and motor evoked potentials (MEPs) are recorded from a muscle using surface electromyography electrodes. TMS may activate, inhibit, or interfere with the activity of various corticocortical and cortico-subcortical networks according to the parameters of stimulation. Stimulation site, coil orientation, biphasic versus monophasic stimulation, stimulus intensity, and frequency can all be used to manipulate the resulting changes to the cerebrum, including how long these changes continue for after stimulation has ceased.

\section{Single- and paired-pulse TMS}

Single-pulse TMS (spTMS) can be applied to the motor cortex to determine motor threshold and generate input-output curves. Motor threshold refers to the lowest TMS intensity required to evoke MEPs in a target muscle in $50 \%$ of trials. Provided spinal motor neuronal excitability is stable, motor threshold is believed to represent a measure of membrane excitability in pyramidal neurons (Ziemann et al., 1996). Single TMS pulses of progressively increasing intensity applied to the motor cortex can be used to generate a recruitment curve. The resulting modulation of MEP amplitude to increasing intensity of TMS pulses appears to provide a measure of excitatory feedback to corticospinal efferent output, which may be glutamatergically mediated (Kaelin-Lang et al., 2002; Prout and Eisen, 1994). By using a paired-pulse TMS paradigm, activation of intracortical inhibitory neurons within the motor cortex is possible (Kujirai et al., 1993). It is suggested that an inhibitory phenomenon takes place at the cortical level (Di Lazzaro et al., 1998) and is the result of the firing of GABAergic interneurons within motor cortex (Boroojerdi et al., 2001; Ziemann et al., 1996). The functional connectivity between the sensory and the primary motor cortex can also be described by TMS. Afferent input (i.e., electrical stimulation of the digital or median nerves) is shown to modify the excitability of the motor cortex induced by TMS with a complex time course (Maertens de Noordhout et al., 1992; Tokimura et al., 2000). This inhibitory phenomenon is termed as "short latency afferent inhibition" and is thought to be regulated by muscarinic cholinergic circuits (Di Lazzaro et al., 2002). Functional connectivity between the two cerebral hemispheres can be studied by transcallosal TMS (Ferbert et al., 1992) by applying a conditioning stimulus to one hemisphere while applying a test stimulus to the other hemisphere. Transcallosal output gives rise to inhibition of the contralateral primary motor cortex (Gerloff et al., 1998; Meyer et al., 1995; Wassermann et al., 1991). 


\section{Repetitive and paired associative TMS}

Single and paired pulses guarantee a high temporal precision (in the milliseconds range). If a train of multiple pulses is applied at a particular frequency, the stimulation is called repetitive TMS (rTMS). In contrast to spTMS, multiple pulses have more prolonged effects on the brain. The nature of the aftereffects depends on the number, intensity, and frequency of stimulation pulses. For example, stimulation at frequencies lower than $1 \mathrm{~Hz}$ reduces cortex excitability (Chen et al., 1997; Romero et al., 2002), while stimulation at frequencies higher than $1 \mathrm{~Hz}$ tends to increase cortical excitability (Berardelli et al., 1998). The duration of such excitability shift depends on the duration of the rTMS exposure, that is, the number of rTMS trains applied and the intertrain interval. Changes in excitability of the neuraxis observed during rTMS appear complex (reviewed in Fitzgerald et al., 2006) and require both studies in humans and in animal models to explore the underlying mechanisms. In contrast, paired associative stimulation has accumulated reasonable evidence for a role of synaptic mechanisms that might relate to long-term potentiation (LTP) and long-term depression (LTD)like effects. Paired associative stimulation was first described by Stefan et al. (2000). The median nerve is activated by bipolar electrical stimulation at the wrist, and spTMS is applied to the hand representation of the contralateral primary motor cortex through a focal figure-of-eight coil. The interstimulus interval is either set to $25 \mathrm{~ms}$ (Stefan et al., 2000) or adjusted to the individual N20latency (plus $2 \mathrm{~ms}$ ) of the median nerve somatosensory evoked potential (Ziemann et al., 2004). LTP-like plasticity, induced by paired associative stimulation, is measured as a long-term increase (>30 min) of the MEP in the target muscle (Stefan et al., 2000) or by an increase in the slope of the MEP intensity curve (Meunier et al., 2007; Rosenkranz et al., 2007). Finally, Huang et al. (2005) have recently described a rapid method to modulate excitability in the motor cortex, termed as "theta burst stimulation." The protocol uses short bursts of low intensity ( $80 \%$ of active motor threshold), high-frequency $(50 \mathrm{~Hz})$ pulses, repeated at $5 \mathrm{~Hz}$-the frequency of the theta rhythm in the electroencephalogram. Epidural recordings suggest that continuous theta burst stimulation has its major effect on the synapse between the interneurones responsible for the indirect $I_{1}$ wave and the corticospinal neurones (Di Lazzaro et al., 2005). Different patterns of delivery of theta burst stimulation (continuous vs. intermittent) produce opposite effects on synaptic efficiency of the stimulated motor cortex (Di Lazzaro et al., 2008; Huang et al., 2005).

\section{TMS in DOC}

Despite widespread use of TMS techniques in neuroscience, very few TMS investigations have been conducted in acute and chronic DOC (reviewed in Lapitskaya et al., 2009). The majority of TMS work with DOC has been undertaken to determine whether TMS has the ability to predict outcome from coma at an early stage or to evaluate whether TMS is able to assess corticospinal motor function more precisely than the clinical examination. No studies have been published so far considering TMS as a tool for investigating pathophysiology aspects of DOC.

\section{The prognostic utility of TMS in coma}

To date four empirical studies have focused on whether TMS can predict outcome in coma. Ying et al. (1992) examined 23 comatose patients (11 traumatic, 12 non-traumatic) within 2-20 days of coma onset and found no relationship between the integrity of MEPs and outcome as measured by the Glasgow Outcome Scale (Jennett and Bond, 1975). Similarly, Facco et al. (1991) investigated 22 comatose patients (13 traumatic, 9 non-traumatic) within 1 week of coma onset and found no relationship between the integrity of MEPs and outcome. A similar finding was also obtained from 30 patients with acute brainstem lesions. Schwarz et al. (2000) used spTMS to record MEPs from a group of patients, whom they 
described as having decreased consciousness (described clinically as comatose, stuporous, and somnolent), having been admitted to the accident and emergency department. However, no correlation was found between MEPs and Glasgow Outcome Scale score 3 months post-ictus. In contrast, Zentner and Rohde (1992) performed spTMS with 39 comatose patients (etiology not available) within 3 days of insult, and found that MEPs presence was weakly correlated with the patients Glasgow Outcome Scale score 3 months and 2 years later.

In summary, TMS studies to date have failed to identify a clear prognostic utility. One of the limitations of the classic MEP in the clinical setting appears to be its absence in some patients with good outcome. A transient absence of MEPs in comatose patients may be due to reversible damage of motor pathways or decreased excitability due to treatment (e.g., sedation, antiepileptic drugs). When cortical excitability is decreased, the single magnetic stimulus might not be strong enough to excite the motor cortex even with the maximal stimulator output; therefore facilitation techniques should be applied in comatose patients. The commonly used technique in healthy volunteers of voluntary muscle contraction is not possible in unresponsive patients thus other techniques (e.g., painful stimulation prior to the TMS; double or repetitive pulse paradigms) have to be used.

\section{The prognostic utility of TMS in VS and MCS}

Patients in VS have "awakened" from their coma (e.g., they open their eyes on stimulation or spontaneously), but remain unaware of self or environment (e.g., they show only reflex motor responses) (Jennett and Plum, 1972). In contrast, patients in MCS show limited but clearly discernible evidence of awareness of self or environment (i.e., reproducible responses to command, pursuit eye movement, etc.). The emergence of MCS is characterized by the recovery of functional communication or use of objects (Giacino et al., 2002). Despite the fact that misdiagnosis of these patients is still frequent in clinical practice (e.g., Schnakers et al., 2006, 2009), the diagnosis of MCS often leads to a better outcome than VS.

To date only three studies have assessed VS and MCS patients with TMS. It should be mentioned that consciousness impairment in the published patient populations is not well defined, as the studies were conducted before the introduction of the MCS criteria (Giacino et al., 2002). Moosavi et al. (1999) applied spTMS to the hand and leg motor area in 19 patients; 6-76 months after severe anoxic brain injury. Eleven patients were consistently unresponsive to simple verbal commands and multimodality sensory stimulation (VS), while eight patients were able to respond with reliable movements such as gaze directed selection of "yes" or "no" signs (MCS). However, none of the patients were able to make isolated finger or thumb movements. Moosavi et al. were unable to elicit MEPs from any muscle in two of the eleven unresponsive, VS patients. Whether the MEPs' absence can be attributed to the focal damage in the respective motor areas remains speculative, as the authors do not present any imaging data on these patients. In the remaining patients, the nonresponsive (VS) patient group differed from the responsive (MCS) patient group in having a higher threshold, longer duration, and greater irregularity in the form of the response, while the threshold, form, and latency of MEPs from the responsive (MCS) group were similar to healthy control subjects.

The second study to investigate VS patients with TMS was conducted by Mazzini et al. (1999), who used TMS to monitor recovery. Mazzini examined MEPs from upper and lower limbs in 27 patients in the subacute period (about 2 months after injury) and then at 6 and 12 months postictus. Patients were either comatose or in the VS at the first examination, while five patients remained VS 1 year after the trauma and one died at the end of the follow-up. During the study period, the authors observed an overall trend toward an increase of amplitude and decrease of latency of MEPs. MEPs from upper and lower limbs progressively normalized in all patients, and at 1 year after trauma, only $12 \%$ of patients had mild abnormalities in MEP responses. The differences between basal MEP scores and those at 
12 months after trauma did not correlate with the Glasgow Coma Scale at the time of injury and the duration of coma. Similarly, no association was found between MEP amplitude or latency at the first examination and outcome, measured by Glasgow Outcome Scale.

Nevertheless, a concomitant increase in MEP amplitude and clinical recovery has been observed in a single case study. Crossley et al. (2005) investigated the relationship between cognitive and behavioral ability and spTMS elicited MEPs. In their case study, recovery from the traumatic coma was monitored using the Wessex Head Injury Matrix (Shiel et al., 2000). Clinical and TMS examinations were performed at 4 weeks post-injury, when the patient showed signs of arousal and alertness (eyes open briefly, attention held momentarily by dominant stimulus), and 12 months later, when the patient was reported to be fully awake and conscious. TMS conducted at 12 months showed an increase in the MEP amplitude in comparison to the recording at 4 weeks, consistent with clinical improvement.

In summary, the results support the idea that a degree of cortical functional integrity is present in post-comatose patients, even in those who are clinically diagnosed as being in a nonresponsive state. Despite the absence of voluntary movements, TMS elicited MEP responses in the majority of severely brain-damaged patients, and a trend toward an increase of amplitude and decrease of latency of MEPs could be observed during the recovery period.

\section{Possible confounding variables influencing TMS results in DOC}

A variety of factors might have effects on the cortical excitability parameters obtained with TMS in DOC patients.

(1) Medication is one of the main pitfalls when interpreting TMS results in DOC patients. TMS is thought to activate the corticospinal neurons transsynaptically (Di Lazzaro et al., 2003), and is more susceptible than transcranial electrical stimulation to inhibitory drug effects, but on the other hand, could have a higher sensitivity to detect superficial, presynaptic lesions. Chronic DOC patients have been weaned off sedation, but many remain on anticonvulsants, antispasticity and analgesic drugs, benzodiazepines, central nervous system stimulants, and antidepressants. While some information has been gathered on how individual drugs influence TMS (reviewed in Paulus et al., 2008), the effects of different combinations of drugs used in severely injured and bedridden patients remains unclear.

(2) Currents induced in the healthy brain by TMS flow parallel to the plane of the stimulation coil, that is, approximately parallel to the brain's cortical surface when the stimulation coil is held tangentially to the scalp (Saypol et al., 1991). This results in preferential activation of neural elements oriented horizontally, that is, parallel to the cortical surface (Amassian et al., 1990; Day et al., 1987). This notion is not unchallenged (Edgley et al., 1990) and is highly dependent on stimulation intensity, coil orientation, sulcal pattern, conductivity of neighboring tissue, and orientation of nerve fibers (Maccabee et al., 1993). In DOC patients, one usually needs higher stimulation intensities to elicit an MEP. The site within the motor system at which decreased excitability occurs, however, is unclear. In the case of severe brain injury, a combination of primary and secondary lesions directly destroy or compress brain tissue and produce local and remote effects on the brain (Plum and Posner, 1983), while diffuse axonal injury causes shearing injuries to the cerebral white matter (Povlishock, 1993). The excitability patterns after brain damage may be as much due to the site and type of damage as to the changes in activity across the undamaged brain.

(3) The amplitude of the MEP is not only dependent on TMS intensity, but is also greatly influenced by factors that affect corticospinal excitability. The excitability of the postsynaptic corticospinal neuron 
may be decreased or increased, possibly relating to changes in extrinsic input to the motor cortex. The magnitude of the intracortical inhibition and facilitation varies depending on the degree of contraction of the target muscle-a critical variable to control for in paired-pulse TMS studies. For example, voluntary contraction of the target muscle enhances excitability at the spinal level and facilitates the responses to TMS (Hess et al., 1986; Thompson et al., 1991). Mental imagery of contraction of the same target muscle results in a similar facilitation of MEPs. Cincotta et al. (1999) reported a case of locked-in syndrome due to a large pontine infarction. One month after the attack, no MEPs could be recorded from either the right upper or lower limb. In contrast, MEPs were obtained from the left hand, although with a prolonged latency and reduced amplitude. When the patient was requested to mentally perform an abduction of her paralyzed left little finger, the latency and the amplitude of these responses improved as compared with the relaxed condition. Although in this patient no control condition was investigated to rule out the possibility that MEP changes might depend upon the patient's arousal level, it seems reasonable to conclude that motor imagery played a major role in determining this facilitation.

(4) The amplitude and the latency of the MEP reflect not only the integrity of the corticospinal tract, but also the excitability of nerve roots and the conduction along the peripheral motor pathway to the muscles. Patients with dysfunction at any level along the corticospinal pathway may show abnormal MEPs, while the presence of intact MEPs suggests integrity of the pyramidal tract. Pronounced lengthening of central motor conduction time suggests demyelination of pathways, while a low-amplitude response, with little delay or absence of response, is more suggestive of loss of neurons or axons. The spectrum of neuromuscular problems in the severely brain injured patient is broad, especially in the acute stage of coma. In the intensive care unit, $70 \%$ of patients with systemic inflammatory response syndrome suffer from critical illness polyneuropathy (Witt et al., 1991). Axonal motor neuropathy and neuromuscular junction dysfunction, due to administration of neuromuscular blocking agents and steroids, myopathy (diffuse, type II muscle fibers atrophy, thick-filament myopathy, necrotizing myopathy), and atrophy of the muscles due to prolonged immobility, might affect both amplitude and latency of MEPs (Zifko et al., 1998).

(5) Maintenance of a constant scalp position with the stimulating coil is critically important in TMS studies because a small change in the position can greatly affect the MEP amplitude. VS patients are, by definition, unable to follow instructions, and adjustment to the robotic coil positioning cannot always be maintained, so TMS experiments often require manual coil positioning, which is difficult over a long session.

In summary, TMS results in DOC should be interpreted with some caution. At present, there is some indication TMS may provide a useful measure of excitability in the targeted cortex and its connections.

\section{Directions for future research}

The last 10 years have been witnessed to important advances in our understanding of DOC. Structural brain imaging studies demonstrate that the behavioral level ultimately achieved by a patient following severe brain injury cannot be simply graded by the degree of diffuse axonal and direct ischemic brain damage (Giacino et al., 2006). Changes in cerebral metabolism and excitability of brain areas remote from a lesion have been reported in animals and humans and implicated as mechanisms relevant for functional recovery (Andrews, 1991; Seitz et al., 1999). The underlying mechanisms involve the unmasking of existing, but latent, horizontal 
connections (Sanes and Donoghue, 2000) or modulation of synaptic efficacy such as LTP or LTD (Cooke and Bliss, 2006). The neurotransmitter systems involved in mediating these effects include the inhibitory GABAergic (Hess et al., 1996b; Hess and Donoghue, 1994) as well as the excitatory glutamatergic system with activation of $\mathrm{N}$-methyl-d-aspartate receptors (Hess et al., 1996a). Single-pulse stimulation paradigms do not seem to provide sufficient information about the integrity of inhibitory and excitatory networks in DOC. However, paired-pulse and repetitive stimulation paradigms might identify signs of preserved brain connectivity in noncommunicative brain-damaged patients. Transcallosal inhibition (Takeuchi et al., 2006) and short latency afferent inhibition (Fujiki et al., 2006) are worthy of further investigation in DOC as they are potent connectivity markers.

In addition, rTMS modulates cortical excitability beyond the duration of the rTMS trains themselves. Particularly tantalizing is the possibility that modulation of cortical excitability by rTMS might have therapeutic applications in DOC conditions. At present, there are no proven treatments for promoting recovery from DOC. Inspiration could be picked up from multifarious studies in major depressive disorders (Fregni et al., 2006; Gross et al., 2007; Pascual-Leone et al., 1998), cognitive (reviewed in Miniussi et al., 2008) and motor (reviewed in Edwards and Fregni, 2008) rehabilitation in stroke, traumatic brain injury, and neurodegenerative disorders. Indeed, in a recent case study, Pape et al. (2009) described the results of a safety and efficacy study that examined a therapeutic rTMS protocol for persons with severe traumatic brain injury. A 6-week rTMS protocol (30 sessions) was delivered to a 26-year-old man who remained in VS 10 months after severe traumatic brain injury. Stimulation was directed over the right dorsolateral prefrontal cortex. Neurobehavioral assessments were obtained at baseline, every fifth rTMS session, and at a 6-week follow-up. There were no adverse events related to the provision of rTMS treatment. A trend toward significant neurobehavioral gains was temporally related to the provision of rTMS. Although it is too early to conclude that rTMS might have any therapeutic application, it is possible rTMS could modify cortical excitability. Indeed, theta burst stimulation paradigms are of particular interest in DOC, as they seem to facilitate long-lasting cortical excitability changes (inhibitory and excitatory) after very short stimulation sessions. Furthermore, noninvasive brain stimulation, integrated with EEG and neuroimaging techniques, may provide a means to investigate a range of stimulation sites (i.e., occipital and frontal areas, precuneus, etc.) and parameters for deep brain stimulation in order to facilitate cognitive recovery (Schiff et al., 2007) in DOC.

Here, we have briefly reviewed some of the ways in which TMS could be applied to evaluate cortical excitability in DOC. Although early empirical studies suggest that TMS has very little prognostic utility, the more recent application of rTMS, particularly theta burst, suggests it may have a therapeutic role, promoting changes in cortical excitability. At present, the field of neurorehabilitation lacks evidence-based treatments for promoting cognitive recovery in DOC, and thus it is hoped more studies utilizing TMS will be seen in the near future.

\section{Acknowledgments}

NL is supported by a research fellowship from the European Neurological Society and the Danish Medical Research Council. OG is research fellow at the Fonds de la Recherche Scientifique (FRS). This work was supported by European Commission (DISCOS, Mindbridge and COST) and James McDonnell Foundation.

\section{References}

Amassian, V. E., Quirk, G. J., \& Stewart, M. (1990). A comparison of corticospinal activation by magnetic coil and electrical stimulation of monkey motor cortex. Electroencephalography and Clinical Neurophysiology, 77, 390-401.

Andrews, R. J. (1991). Transhemispheric diaschisis. A review and comment. Stroke, 22, 943-949.

Barker, A. T., \& Jalinous, R. (1985). Non-invasive magnetic stimulation of human motor cortex. Lancet, 1, 1106-1107. 
Berardelli, A., Inghilleri, M., Rothwell, J. C., Romeo, S., Curra, A., Gilio, F., et al. (1998). Facilitation of muscle evoked responses after repetitive cortical stimulation in man. Experimental Brain Research, 122, 79-84.

Boroojerdi, B., Battaglia, F., Muellbacher, W., \& Cohen, L. G. (2001). Mechanisms influencing stimulus-response properties of the human corticospinal system. Clinical Neurophysiology, 112, 931-937.

Chen, R., Classen, J., Gerloff, C., Celnik, P., Wassermann, E. M., Hallett, M., et al. (1997). Depression of motor cortex excitability by low-frequency transcranial magnetic stimulation. Neurology, 48, 1398-1403.

Cincotta, M., Tozzi, F., Zaccara, G., Borgheresi, A., Lori, S., Cosottini, M., et al. (1999). Motor imagery in a locked-in patient: Evidence from transcranial magnetic stimulation. Italian Journal of Neurological Sciences, 20, 37-41.

Cooke, S. F., \& Bliss, T. V. P. (2006). Plasticity in the human central nervous system. Brain, 129, 1659-1673.

Crossley, M., Shiel, A., Wilson, B., Coleman, M. R., Gelling, L., Fryer, T., et al. (2005). Monitoring emergence from coma following severe brain injury in an octogenarian using behavioural indicators, electrophysiological measures and metabolic studies: A demonstration of the potential for good recovery in older adults. Brain Injury, 19, 729-737.

Day, B. L., Thompson, P. D., Dick, J. P., Nakashima, K., \& Marsden, C. D. (1987). Different sites of action of electrical and magnetic stimulation of the human brain. Neuroscience Letters, 75, 101-106.

Di Lazzaro, V., Oliviero, A., Pilato, F., Mazzone, P., Insola, A., Ranieri, F., et al. (2003). Corticospinal volleys evoked by transcranial stimulation of the brain in conscious humans. Neurological Research, 25, 143-150.

Di Lazzaro, V., Oliviero, A., Pilato, F., Saturno, E., Dileone, M., Mazzone, P., et al. (2004). The physiological basis of transcranial motor cortex stimulation in conscious humans. Clinical Neurophysiology, 115, 255-266.

Di Lazzaro, V., Oliviero, A., Profice, P., Saturno, E., Pilato, F., Insola, A., et al. (1998). Comparison of descending volleys evoked by transcranial magnetic and electric stimulation in conscious humans. Electroencephalography and Clinical Neurophysiology, 109, 397-401.

Di Lazzaro, V., Oliviero, A., Tonali, P. A., Marra, C., Daniele, A., Profice, P., et al. (2002). Noninvasive in vivo assessment of cholinergic cortical circuits in AD using transcranial magnetic stimulation. Neurology, 59, 392-397.

Di Lazzaro, V., Pilato, F., Dileone, M., Profice, P., Oliviero, A., Mazzone, P., et al. (2008). The physiological basis of the effects of intermittent theta burst stimulation of the human motor cortex. Journal of Physiology (London), 586, 3871-3879.

Di Lazzaro, V., Pilato, F., Saturno, E., Oliviero, A., Dileone, M., Mazzone, P., et al. (2005). Theta-burst repetitive transcranial magnetic stimulation suppresses specific excitatory circuits in the human motor cortex. Journal of Physiology (London), 565, 945-950.

Edgley, S. A., Eyre, J. A., Lemon, R. N., \& Miller, S. (1990). Excitation of the corticospinal tract by electromagnetic and electrical stimulation of the scalp in the macaque monkey. Journal of Physiology (London), 425, 301-320.

Edwards, D., \& Fregni, F. (2008). Modulating the healthy and affected motor cortex with repetitive transcranial magnetic stimulation in stroke: Development of new strategies for neurorehabilitation. NeuroRehabilitation, 23, 3-14.

Facco, E., Baratto, F., Munari, M., Dona, B., Casartelli Liviero, M., Behr, A. U., et al. (1991). Sensorimotor central conduction time in comatose patients. Electroencephalography and Clinical Neurophysiology, 80, 469-476.

Ferbert, A., Priori, A., Rothwell, J. C., Day, B. L., Colebatch, J. G., \& Marsden, C. D. (1992). Interhemispheric inhibition of the human motor cortex. Journal of Physiology (London), 453, 525-546.

Fitzgerald, P. B., Fountain, S., \& Daskalakis, Z. J. (2006). A comprehensive review of the effects of rTMS on motor cortical excitability and inhibition. Clinical Neurophysiology, 117, 2584-2596.

Fregni, F., Boggio, P. S., Nitsche, M. A., Marcolin, M. A., Rigonatti, S. P., \& Pascual-Leone, A. (2006). Treatment of major depression with transcranial direct current stimulation. Bipolar Disorders, 8, 203-204.

Fujiki, M., Hikawa, T., Abe, T., Ishii, K., \& Kobayashi, H. (2006). Reduced short latency afferent inhibition in diffuse axonal injury patients with memory impairment. Neuroscience Letters, 405, 226-230.

Gerloff, C., Cohen, L. G., Floeter, M. K., Chen, R., Corwell, B., \& Hallett, M. (1998). Inhibitory influence of the ipsilateral motor cortex on responses to stimulation of the human cortex and pyramidal tract. Journal of Physiology (London), 510, 249-259.

Giacino, J. T., Ashwal, S., Childs, N., Cranford, R., Jennett, B., Katz, D. I., et al. (2002). The minimally conscious state: Definition and diagnostic criteria. Neurology, 58, 349-353.

Giacino, J. T., Hirsch, J., Schiff, N., \& Laureys, S. (2006). Functional neuroimaging applications for assessment and rehabilitation planning in patients with disorders of consciousness. Archives of Physical Medicine and Rehabilitation, 87, S67-S76.

Gross, M., Nakamura, L., Pascual-Leone, A., \& Fregni, F. (2007). Has repetitive transcranial magnetic stimulation (rTMS) treatment for depression improved? A systematic review and meta-analysis comparing the recent vs. the earlier rTMS studies. Acta Psychiatrica Scandinavica, 116, 165-173.

Hess, C. W., Mills, K. R., \& Murray, N. M. F. (1986). Magnetic stimulation of the human brain-facilitation of motor responses by voluntary contraction of ipsilateral and contralateral muscles with additional observations on an amputee. Neuroscience Letters, 71, 235-240.

Hess, G., Aizenman, C. D., \& Donoghue, J. P. (1996a). Conditions for the induction of long-term potentiation in layer II/III horizontal connections of the rat motor cortex. Journal of Neurophysiology, 75, 1765-1778.

Hess, G., Aizenman, C. D., \& Donoghue, J. P. (1996b). Conditions for the induction of long-term potentiation in layer II/III horizontal connections of the rat motor cortex. Journal of Neurophysiology, 75, 1765-1778. 
Hess, G., \& Donoghue, J. P. (1994). Long-term potentiation of horizontal connections provides a mechanism to reorganize cortical motor maps. Journal of Neurophysiology, 71, 2543-2547.

Huang, Y. Z., Edwards, M. J., Rounis, E., Bhatia, K. P., \& Rothwell, J. C. (2005). Theta burst stimulation of the human motor cortex. Neuron, 45, 201-206.

Jennett, B., \& Bond, M. (1975). Assessment of outcome after severe brain damage. Lancet, 1, 480-484.

Jennett, B., \& Plum, F. (1972). Persistent vegetative state after brain damage. A syndrome in search of a name. Lancet, 1, 734-737.

Kaelin-Lang, A., Luft, A. R., Sawaki, L., Burstein, A. H., Sohn, Y. H., \& Cohen, L. G. (2002). Modulation of human corticomotor excitability by somatosensory input. Journal of Physiology (London), 540, 623-633.

Kujirai, T., Caramia, M. D., Rothwell, J. C., Day, B. L., Thompson, P. D., Ferbert, A., et al. (1993). Corticocortical inhibition in human motor cortex. Journal of Physiology (London), 471, 501-519.

Lapitskaya, N., Delvaux, V., Overgaard, M., Nielsen, F., Maertens de Noordhout, A., Moonen, G., et al. (2009). Transcranial magnetic stimulation in disorders of consciousness. Reviews in the Neurosciences, 20 (3-4), in press.

Maccabee, P. J., Amassian, V. E., Eberle, L. P., \& Cracco, R. Q. (1993). Magnetic coil stimulation of straight and bent amphibian and mammalian peripheral nerve in vitro-Locus of excitation. Journal of Physiology (London), 460, 201-219.

Maertens de Noordhout, A., Rothwell, J. C., Day, B. L., Dressler, D., Nakashima, K., Thompson, P. D., et al. (1992). Effect of digital nerve stimuli on responses to electrical or magnetic stimulation of the human brain. Journal of Physiology (London), 447, 535-548.

Mazzini, L., Pisano, F., Zaccala, M., Miscio, G., Gareri, F., \& Galante, M. (1999). Somatosensory and motor evoked potentials at different stages of recovery from severe traumatic brain injury. Archives of Physical Medicine and Rehabilitation, 80, 33-39.

Meunier, S., Russmann, H., Simonetta-Moreau, M., \& Hallett, M. (2007). Changes in spinal excitability after PAS. Journal of Neurophysiology, 97, 3131-3135.

Meyer, B. U., Roricht, S., Voneinsiedel, H. G., Kruggel, F., \& Weindl, A. (1995). Inhibitory and excitatory interhemispheric transfers between motor cortical areas in normal humans and patients with abnormalities of the corpus callosum. Brain, 118, 429-440.

Miniussi, C., Cappa, S. F., Cohen, L. G., Floel, A., Fregni, F., Nitsche, M. A., et al. (2008). Efficacy of repetitive transcranial magnetic stimulation/transcranial direct current stimulation in cognitive neurorehabilitation. Brain Stimulation, 1, 326-336.

Moosavi, S. H., Ellaway, P. H., Catley, M., Stokes, M. J., \& Haque, N. (1999). Corticospinal function in severe brain injury assessed using magnetic stimulation of the motor cortex in man. Journal of Neurological Sciences, 164, 179-186.

Pape, T., Rosenow, J., Lewis, G., Ahmed, G., Walker, M., Guernon, A., et al. (2009). Repetitive transcranial magnetic stimulation-associated neurobehavioral gains during coma recovery. Brain Stimulation, 2, 22-35.

Pascual-Leone, A., Tormos, J. M., Keenan, J., Tarazona, F., Canete, C., \& Catala, M. D. (1998). Study and modulation of human cortical excitability with transcranial magnetic stimulation. Journal of Clinical Neurophysiology, 15, 333-343.

Paulus, W., Classen, J., Cohen, L. G., Large, C. H., Di Lazzaro, V., Nitsche, M., et al. (2008). State of the art: Pharmacologic effects on cortical excitability measures tested by transcranial magnetic stimulation. Brain Stimulation, 1, 151-163.

Plum, F., \& Posner, J. B. (1983). The diagnosis of stupor and coma. Philadelphia, PA: Davis FA.

Povlishock, J. T. (1993). Pathobiology of traumatically induced axonal injury in animals and man. Annals of Emergency Medicine, 22, 980-986.

Prout, A. J., \& Eisen, A. A. (1994). The cortical silent period and amyotrophic lateral sclerosis. Muscle and Nerve, 17, 217-223.

Romero, J. R., Anschel, D., Sparing, R., Gangitano, M., \& Pascual-Leone, A. (2002). Subthreshold low frequency repetitive transcranial magnetic stimulation selectively decreases facilitation in the motor cortex. Clinical Neurophysiology, 113, 101-107.

Rosenkranz, K., Kacar, A., \& Rothwell, J. C. (2007). Differential modulation of motor cortical plasticity and excitability in early and late phases of human motor learning. Journal of Neuroscience, 27, 12058-12066.

Sanes, J. N., \& Donoghue, J. P. (2000). Plasticity and primary motor cortex. Annual Review of Neuroscience, 23, 393-415.

Saypol, J. M., Roth, B. J., Cohen, L. G., \& Hallett, M. (1991). A theoretical comparison of electric and magnetic stimulation of the brain. Annals of Biomedical Engineering, 19, 317-328.

Schiff, N. D., Giacino, J. T., Kalmar, K., Victor, J. D., Baker, K., Gerber, M., et al. (2007). Behavioural improvements with thalamic stimulation after severe traumatic brain injury. Nature, 448, 600-603.

Schnakers, C., Giacino, J., Kalmar, K., Piret, S., Lopez, E., Boly, M., et al. (2006). Does the FOUR score correctly diagnose the vegetative and minimally conscious states? Annals of Neurology, 60, 744-745.

Schnakers, C., Vanhaudenhuyse, A., Giacino, J., Ventura, M., Boly, M., Majerus, S., Moonen, G., et al. (2009). Diagnostic accuracy of the vegetative and minimally conscious state: Clinical consensus versus standardized neurobehavioral assessment. BMC Neurology, 9 (July 21), 35.

Schwarz, S., Hacke, W., \& Schwab, S. (2000). Magnetic evoked potentials in neurocritical care patients with acute brainstem lesions. Journal of Neurological Sciences, 172, 30-37.

Seitz, R. J., Azari, N. P., Knorr, U., Binkofski, F., Herzog, H., \& Freund, H. J. (1999). The role of diaschisis in stroke recovery. Stroke, 30, 1844-1850.

Shiel, A., Horn, S. A., Wilson, B. A., Watson, M. J., Campbell, M. J., \& McLellan, D. L. (2000). The wessex head injury matrix (WHIM) main scale: A preliminary report on a scale to assess and monitor patient recovery after severe head injury. Clinical Rehabilitation, 14, 408-416. 
Siebner, H. R., \& Rothwell, J. (2003). Transcranial magnetic stimulation: New insights into representational cortical plasticity. Experimental Brain Research, 148, 1-16.

Stefan, K., Kunesch, E., Cohen, L. G., Benecke, R., \& Classen, J. (2000). Induction of plasticity in the human motor cortex by paired associative stimulation. Brain, 123, 572-584.

Takeuchi, N., Ikoma, K., Chuma, T., \& Matsuo, Y. (2006). Measurement of transcallosal inhibition in traumatic brain injury by transcranial magnetic stimulation. Brain Injury, 20, 991-996.

Thompson, P. D., Day, B. L., Crockard, H. A., Calder, I., Murray, N. M. F., Rothwell, J. C., et al. (1991). Intraoperative recordings of motor tract potentials at the cervicomedullary junction following scalp electrical and magnetic stimulation of the motor cortex. Journal of Neurology, Neurosurgery, and Psychiatry, 54, 618-623.

Tokimura, H., Di Lazzaro, V., Tokimura, Y., Oliviero, A., Profice, P., Insola, A., et al. (2000). Short latency inhibition of human hand motor cortex by somatosensory input from the hand. Journal of Physiology (London), 523, 503-513.

Wassermann, E. M., Fuhr, P., Cohen, L. G., \& Hallett, M. (1991). Effects of transcranial magnetic stimulation on ipsilateral muscles. Neurology, 41, 1795-1799.
Witt, N. J., Zochodne, D. W., Bolton, C. F., Maison, F. G., Wells, G., Young, G. B., et al. (1991). Peripheral nerve function in sepsis and multiple organ failure. Chest, 99, 176-184.

Ying, Z., Schmid, U. D., Schmid, J., \& Hess, C. W. (1992). Motor and somatosensory evoked potentials in coma: Analysis and relation to clinical status and outcome. Journal of Neurology, Neurosurgery, and Psychiatry, 55, 470-474.

Zentner, J., \& Rohde, V. (1992). The prognostic value of somatosensory and motor evoked potentials in comatose patients. Neurosurgery, 31, 429-434.

Ziemann, U., Iliac, T. V., Pauli, C., Meintzschel, F., \& Ruge, D. (2004). Learning modifies subsequent induction of long-term potentiation-like and long-term depression-like plasticity in human motor cortex. Journal of Neuroscience, 24, $1666-1672$.

Ziemann, U., Lonnecker, S., Steinhoff, B. J., \& Paulus, W. (1996). Effects of antiepileptic drugs on motor cortex excitability in humans: a transcranial magnetic stimulation study. Annals of Neurology, 40, 367-378.

Zifko, U. A., Zipko, H. T., \& Bolton, C. F. (1998). Clinical and electrophysiological findings in critical illness polyneuropathy. Journal of Neurological Sciences, 159, 186-193. 